\title{
Lateral masking as a function of spacing
}

\author{
GEORGE WOLFORD and LAWRENCE CHAMBERS \\ Dartmouth College, Hanover, New Hampshire
}

\begin{abstract}
It is argued that lateral masking is a composite of several processes. These processes include response competition, distribution of attention, perceptual grouping, and feature (contour) interaction. Three experiments were carried out in an attempt to isolate some of the components. In the first two experiments, it was shown that feature interaction dominates at close spacing but other processes dominate at wider spacing. The third experiment showed that at least part of the effect of perceptual grouping appears to be information provided about target location.
\end{abstract}

The probability of correctly identifying a target (e.g., a letter) is generally reduced when that target is surrounded by other items. This effect, referred to as lateral masking, has been demonstrated by a number of investigators (e.g., Bouma, 1970; Mackworth, 1965; Wolford \& Hollingsworth, 1974). The purpose of the present manuscript is to argue that lateral masking is a composite of several factors. We begin by summarizing some properties of lateral masking, then present some of the possible components of the effect, and, finally, describe some experiments that were designed to isolate the components.

Several properties of lateral masking seem to be fairly well established. (1) Lateral masking is more pronounced in the periphery than in the center of the fovea (Bouma, 1970; Kolers \& Rosner, 1960; Matthews, 1973; Wolford \& Hollingsworth, 1974). (2) The effect of a mask is diminished as the space between the target and mask is increased (B. A. Eriksen \& C. W. Eriksen, 1974; C. W. Eriksen \& Rohrbaugh, 1970; Flom, Weymouth, \& Kahneman, 1963). (3) Lateral masking tends to be asymmetric in that a mask placed on the peripheral side of a target is more effective than a mask placed on the foveal side (Banks, Larson, \& Prinzmetal, 1979; Bouma, 1973; Chastain \& Lawson, 1979; Mackworth, 1965; Wolford \& Hollingsworth, 1974).

A number of different models or constructs have been proposed to account for lateral masking. Probably because the properties of lateral masking listed above appear sensory in nature, many models assume that lateral masking results from the interaction of target and mask at the featural level (Bjork \& Murray, 1977; Estes, 1972; Krumhansl, 1977; Wolford, 1975).

This research was supported, in part, by NIMH Grant MH 33179. The article was written while the first author was visiting the MRC Applied Psychology Unit in Cambridge, England. We appreciate their generous support. We would like to thank William Banks for convincing us of the need to do the rating studies. We also wish to thank John Duncan for his helpful comments on the manuscript. Our mailing address is: Department of Psychology, Dartmouth College, Hanover, New Hampshire 03755.
Two of the models are outlined to illustrate featureinteraction explanations.

Estes (1972) proposed the interactive-channels model, in which two nearby items are presumed to compete for a limited set of feature detectors. Assumptions about the retinal distribution of feature detectors permit the model to account for the first two properties listed earlier. The third property (asymmetry) is not dealt with in any direct way.

Wolford (1975) proposed the perturbation model in which the features of a character drift over time. Lateral masking occurs when the features of the mask combine with the features of the target, leading to a failure of identification. Assumptions concerning the nature and direction of the perturbation process permit the model to account for the properties listed above.

These feature-interaction models, then, provide an account of lateral masking and many of its properties. The questions of concern are whether there are other properties of lateral masking not captured by the feature-interaction models, and, perhaps more importantly, whether some other construct might provide a more accurate account of even those properties listed earlier.

\section{Response Competition}

C. W. Eriksen and his colleagues have argued that many of the effects of lateral masking are the result of response competition rather than feature interaction (e.g., B. A. Eriksen \& C. W. Eriksen, 1974; C. W. Eriksen \& Hoffman, 1973; C. W. Eriksen \& Schultz, 1978). Many of their claims are based on paradigms using two response sets. The basic finding is that performance in identifying a target is degraded if the target is surrounded by letters of the opposite response set but facilitated if the target is surrounded by letters of the same response set.

The current feature-interaction models cannot deal with the effects of response compatibility. The models would require the addition of new processes to account for those effects. The next question is whether 
the notion of response competition is sufficient to account for lateral masking. There are a couple of lines of evidence which suggest that the answer is no. First, the degradation and facilitation due to incompatible and compatible masks were relative to performance on strings in which the target was surrounded by neutral letters. The presence of any surrounding letters, even identical or compatible, led to lower performance than was obtained when the target was presented alone (B. A. Eriksen \& C. W. Eriksen, 1974; C. W. Eriksen \& Hoffman, 1973). Second, items from domains different from that of the target often lead to as much masking as items from the same domain. C. W. Eriksen and Rohrbaugh (1970) found that small disks were about as effective as letters at masking target letters. C. W. Eriksen and Schultz (1978) suggest that contour interaction is probably a part of lateral masking at close spacing.

\section{Attention and Perceptual Grouping}

Several investigators have argued that the interaction between simultaneous items is better understood in terms of perceptual grouping and/or distribution of attention than in terms of interaction at the featural level (Banks, Larson, \& Prinzmetal, 1979; Kahneman \& Henik, 1977; Prinzmetal, 1981; Treisman \& Schmidt, 1982). Those investigators demonstrated several phenomena which cannot be handled by current feature-interaction models. Again, however, we are interested in determining whether perceptual grouping and/or distribution of attention are sufficient constructs for explaining all of the effects associated with lateral masking. In searching for an answer to this question, we were struck by the fact that the studies cited in this section tend to use rather wide spacing between characters (generally a degree or more), while the experiments in support of feature models tend to use close spacing between characters. The possibility exists, then, that qualitatively different processes are involved at different spacings. To explore this possibility, we examined the effects reported by Kahneman et al. and Banks et al. as a function of intercharacter spacing.

Kahneman and Henik (1977) used a paradigm in which they presented subjects briefly with a series of digits divided into two groups (e.g., four or five digits separated from three or two digits by a space). Their typical finding was that performance on items within a group was quite constant but that performance levels between groups were quite different (see Table 1). They proposed an explanation based on the distribution of attention. Attention was assumed to be directed initially to one of the groups assuring relatively high performance on that group's members. Only after the first group was processed was the second group attended to; and by that time, the second group might have faded from memory.

The layout of Table 1 requires some explanation. The data in Row 1 are from a display containing a group of four characters separated from a group of two characters. The other three rows are from displays containing five and two groupings. The percentages represent the accuracy of report from each position. The " $x$ "'s in Row 2 appear because the data are not available in Kahneman et al. Rows 1-3 represent displays presented centered around the fixation point. Row 4 represents a display presented in the right visual field.

Kahneman et al. discounted a featural explanation of their results based on several considerations. The first consideration was the uniformity of performance within a group. The perturbation model, for instance, predicts a serial position curve within groups. Kahneman et al. did find a modest serial position effect with a five and two grouping (see Table 1). They attributed this to short-term memory. The second consideration was the similarity of their results across various interletter spacing conditions. They more than doubled spacing with little or no effect on performance. As mentioned previously, the featureinteraction models predict an effect of spacing.

The intercharacter spacings used by Kahneman et al., however, were quite wide by some standards. Their closest spacing was $.52 \mathrm{deg}$ between letter centers, and their most distant spacing was $1.18 \mathrm{deg}$. The displays of Kahneman et al. were centered on the fovea. It is possible that $.52 \mathrm{deg}$ is outside the limit of feature interpretation in this region of the retina (Flom, Weymouth, \& Kahneman, 1963).

Data from a previous experiment allow us to examine this possibility. Some of the displays in Wolford and Hollingsworth (1974) are comparable to those used by Kahneman et al., only with closer spacing. Among other conditions, Wolford et al. presented

Table 1

Comparison of Whole-Report Performance as a Function of Spacing + (Fixation)

\begin{tabular}{|c|c|c|c|c|c|c|c|c|c|}
\hline \multirow[t]{2}{*}{ Distant* } & \multicolumn{2}{|c|}{93} & 83 & \multicolumn{2}{|c|}{86} & \multicolumn{2}{|c|}{$29 \quad 39$} & & \\
\hline & 93 & 86 & 84 & 71 & 66 & $\mathrm{X}$ & $\mathrm{X}$ & & \\
\hline Close** & 97 & 80 & 64 & 59 & $\begin{array}{l}54 \\
99\end{array}$ & $93^{20} 70$ & $\begin{array}{r}34 \\
49\end{array}$ & 55 & 12 \\
\hline
\end{tabular}

Note-See text for explanation. *From Kahneman and Henik (1977). **From Wolford and Hollingsworth (1974). 
strings containing a group of five randomly chosen letters and a group of two randomly chosen letters with the two groups separated in space. The spacing between letter centers within a group was $.21 \mathrm{deg}$.

The data from two such display conditions, presented at different retinal locations, are shown in Table 1 along with comparison data from Kahneman et al. The data from our displays show a much steeper serial position curve. Comparing Rows 2 and 3 of Table 1, Kahneman et al. show a drop of .27 across the five-letter group. We show a drop of .43. When the fifth letter is centered on the fovea, there was no upswing in performance between the fourth and fifth letter (see Rows 2 and 3 of Table 1). There was an upswing for the display located further into the periphery (see Row 4 of Table 1). The steep serial position curve and presence or absence of upswings at various retinal locations are all predicted by the perturbation model (Wolford, 1975) but seem outside the scope of a model based on the distribution of attention alone. The grouping effects found in Kahneman et al. at wider spacing, however, are not readily predicted by feature interaction alone, but seem quite consistent with a model based on the distribution of attention.

Banks, Larson, and Prinzmetal (1979, Experiment 3) presented data which are in sharp contradiction to the predictions of a feature-interaction model. They presented subjects with a single target located 5 deg into the periphery. The target was flanked on one side either by a single mask (the letter $\mathrm{H}$ ) or by a column of five masks. The surprising finding was that performance was actually better in the five-mask condition than in the single-mask condition. This result is contrary to a feature-interaction model, since the presence of more masks should lead to more feature interaction and lower performance. Banks et al. explained the result by proposing that the column of masks forms a unified group which perceptually isolates the target from the masks.

Once again, however, the spacing used by Banks et al. was quite generous. The separation of target and mask(s) was $1 \mathrm{deg}$ in their experiment. As in the Kahneman et al. example above, it is possible that different results would be obtained at close and distant spacing. The purpose of the first experiment, therefore, was to replicate the third experiment of Banks et al., both at the spacing they used and at closer spacing.

\section{EXPERIMENT 1A}

The first two experiments are divided into two parts. The first part is the actual letter-detection task. In the second part of each experiment, subjects made judgments as to the perceived groupings in each of the displays. In Experiment 1, different subjects participated in the two phases. In Experiment 2, some subjects participated in both parts and some subjects only in the second part.

\section{Method}

Subjects. Eight Dartmouth students served as subjects. All had normal or corrected-to-normal vision.

Design. As nearly as possible, the first experiment was a replication of Banks et al. (1979, Experiment 3) at the spacing used in their experiment (namely, mask and target separated by $1.0 \mathrm{deg}$ ) and a second replication in which the target and mask were separated by $.3 \mathrm{deg}$ in all conditions. There were, then, four orthogonal variables in this experiment: the three used by Banks et al. (i.e., a peripheral vs. central placement of masks relative to the target, left visual field vs. right visual field, and single vs. grouped mask) and a fourth variable, close vs. distant spacing.

Stimuli and Apparatus. Stimuli were presented on a video monitor controlled by a Terak 8510 minicomputer, viewed at a distance of $71 \mathrm{~cm}$. Viewing distance was controlled by a wooden hood with eyepiece, attached to the front of the monitor. The letters were standard Terak software characters: uppercase $A, Y, U$, and $T$ as targets and uppercase $H$ s as masks. These letters were $.3 \mathrm{deg}$ high and $.2 \mathrm{deg}$ wide; Banks et al. used .4 and $.3 \mathrm{deg}$ of height and width. A plus sign centered on the screen served as the fixation point. All characters were shown as black characters on a white screen. Selected keys on the Terak keyboard served as the response panel.

All targets were presented 5.23 deg to the left or right of the fixation point on the same horizontal axis as the fixation point. Masks were also centered on this axis. Figure 1 shows the stimulus configurations used. In Figures 1 and 3 the letter $T$ refers to target and the letter $M$ to mask. The group mask was a column of $\mathrm{Hs}$, with the middle $H$ on the same horizontal axis as the target and with two other $\mathrm{Hs}$ above and two below the plane of the target. The Hs were separated by $.51 \mathrm{deg}$ vertically, center to center. Single masks consisted of a single $\mathbf{H}$ on the same horizontal axis as the target. Masks appeared either .30 or $.96 \mathrm{deg}$ away from the target, center to center.

Procedure. Each subject participated in four sessions. Each session contained eight blocks. The type of mask (single vs. group) and location of mask relative to target were held constant within a block. There were four types of blocks: group central, group peripheral, single central, and single peripheral. Two of each block type were shown during each session. Each block contained 64

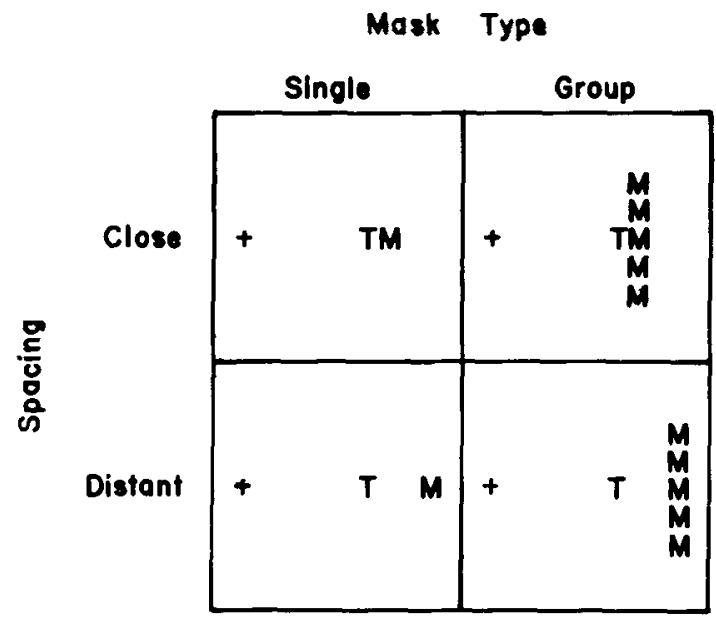

Figure 1. Stimulus configurations for Experiment 1. 
trials ( 4 targets $\times 2$ visual fields $\times 2$ target mask separations $\times$ 4 repetitions). The order of blocks was randomized within and across sessions, as was the order of trials within a block. The grouping arrangements for a block were described to the subjects at the beginning of each block. The number of trials, blocks, and sessions was chosen so that we would have the same number of data points per condition as Banks et al.

The subjects initiated each trial with a keypress. Following the keypress, the fixation cross appeared and stayed in view for $2 \mathrm{sec}$. The target and mask combination was then shown for $100 \mathrm{msec}$. The subject was instructed to respond with one of the four possible target letters on each trial by pressing the appropriate key.

Each subject's first session began with an additional 256 practice trials, using all of the possible target and mask types. Stimulus duration was reduced over practice blocks from 200 to $100 \mathrm{msec}$.

\section{Results}

Letter detection accuracy in terms of percentage correct was computed for each subject. These scores were analyzed by a five-factor repeated-measures ANOVA. The subjects were more accurate when the mask and target were widely spaced than when they were closely spaced, $66.8 \%$ vs. $52.1 \%[F(1,7)=24.53$, $\mathrm{p}<.0025]$. The main effect of target letters was significant $[F(3,21)=33.30, p<.0001]$, reflecting the higher performance on Ts relative to the other letters. The differences in performance for the main effects of the other three variables did not approach statistical reliability (all Fs $<1$ ).

The primary interest in this experiment concerns letter-detection accuracy as a function of the interaction of mask type and target-mask spacing. The data for this interaction are shown in Figure 2. This interaction was highly reliable $[F(1,7)=33.43, p<.0005]$. The group mask did improve subjects' letter-detection accuracy relative to the single mask as distant spacing, replicating Banks et al. At close spacing, however,

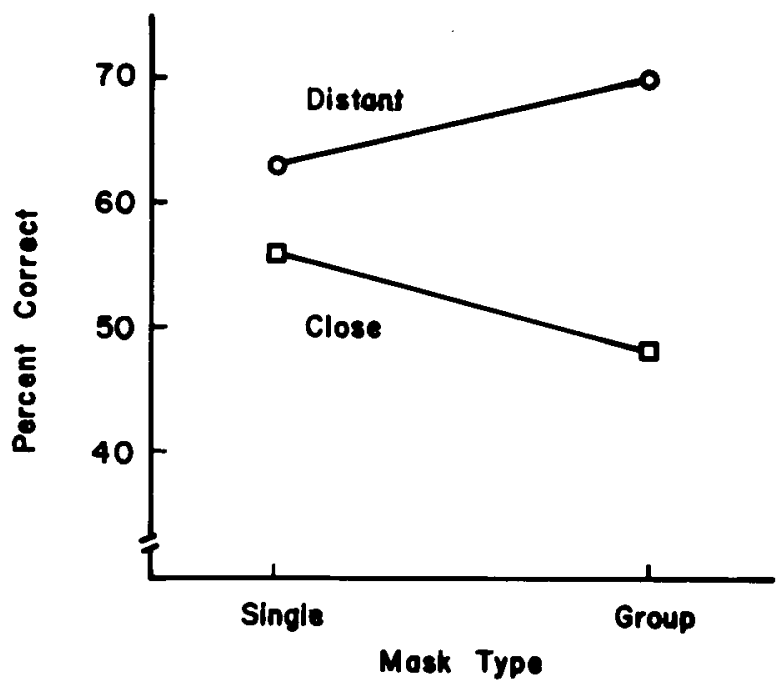

Figure 2. The interaction of mask type and spacing in Experiment 1. the group mask hindered performance relative to the single mask.

Other effects included a significant central-peripheral mask $\times$ visual field interaction $[F(1,7)=25.80, p<$ .0025 ]. Central masks were more effective in the right visual field than in the left visual field, whereas performance on peripheral masks was not affected by visual field. Target-mask spacing interacted with the factor of target letter $[F(3,21)=5.21, p<.01]$. The accuracy of $A, Y$, and $T$ increased in varying amounts with distant spacing, whereas the accuracy for $U$ did not change.

Unlike the finding of Banks et al., the interaction of central vs. peripheral and single vs. group mask did not approach significance. The interaction of central vs. peripheral and spacing did approach significance $[F(1,7)=3.25, p=.11]$. Peripheral masks led to lower performance than central masks in the close spacing conditions ( $51 \%$ vs. $53 \%$ ), but the opposite was true with distant spacing $(67 \%$ vs. $66 \%)$.

The absolute performance level for our subjects at distant spacing was slightly higher than in Banks et al. (67\% for our subjects and $57 \%$ for their subjects). Because of the extra factor (spacing), however, our subjects had twice as many trials. The difference, then, may reflect the extra practice.

\section{EXPERIMENT 1B}

Experiment 1A confirmed the grouping effect at distant spacing. At close spacing, however, adding to the number of masks deteriorated performance, which supports a feature-interaction hypothesis.

One possible problem is that subjects might have had a tendency to group the target with either single or group masks more often at close spacing than at distant spacing, since proximity is also a determiner of grouping. We tested this possibility by having a new group of subjects rate these stimuli on their perceived groupings. This was not done in Banks et al.'s Experiment 3; rather, the groupings were decided a priori based on Gestalt principles. However, these principles do not necessarily lead to clear predictions with close spacing. In addition, the normative grouping with unlimited viewing time may not reflect perceived grouping in a brief display. We therefore had subjects make their ratings using the same viewing conditions as in Experiment $1 \mathrm{~A}$.

\footnotetext{
Method

Subjects. Twenty-four Dartmouth students participated in the experiment for course credit.

Stimuli. The stimuli and apparatus were the same as in Experiment $1 \mathrm{~A}$.

Procedure. Our interest centered around the perceived groupings of four stimulus types (group vs. single mask at near vs. distant spacing). Each subject saw each of the stimulus types twice; once with the mask peripheral to the target and once with the mask central to the target.
} 
Table 2

Ratings of Grouping for the Stimuli Used in Experiments 1 and 2

\begin{tabular}{|c|c|c|}
\hline & $\begin{array}{c}\text { Target } \\
\text { Grouped } \\
\text { With Mask }\end{array}$ & $\begin{array}{l}\text { Target } \\
\text { Sepa- } \\
\text { rately }\end{array}$ \\
\hline \multicolumn{3}{|l|}{ Experiment 1} \\
\hline $\begin{array}{l}\text { Close Spacing } \\
\text { Single Mask } \\
\text { Five Masks }\end{array}$ & $\begin{array}{l}91 \% \\
71 \%\end{array}$ & $\begin{array}{r}9 \% \\
29 \%\end{array}$ \\
\hline $\begin{array}{l}\text { Distant Spacing } \\
\text { Single Mask } \\
\text { Five Masks }\end{array}$ & $\begin{array}{l}13 \% \\
20 \%\end{array}$ & $\begin{array}{l}87 \% \\
80 \%\end{array}$ \\
\hline Experiment 2 & & \\
\hline $\begin{array}{l}\text { Wing } \\
\text { Subjects in Detection Experiment } \\
\text { New Subjects }\end{array}$ & $\begin{array}{l}65 \% \\
59 \%\end{array}$ & $\begin{array}{l}35 \% \\
41 \%\end{array}$ \\
\hline $\begin{array}{l}\text { Column } \\
\text { Subjects in Detection Experiment } \\
\text { New Subjects }\end{array}$ & $\begin{array}{l}57 \% \\
54 \%\end{array}$ & $\begin{array}{l}43 \% \\
46 \%\end{array}$ \\
\hline
\end{tabular}

The rating procedure was similar to that used by Banks and Prinzmetal (1976). The subjects were shown printed examples of all of the stimulus types and instructed that their task was to determine whether any of the letters appeared to group together. They were given sheets of paper, each sheet containing a fixation cross. After viewing each stimulus, they were asked to draw on a card the location and nature of the target and mask and then to circle any letters that appeared to group together. They were permitted to use as many groups as they wished, subject to the constraint that the groups be disjoint. To further clarify the instructions, the experimenter demonstrated all of the ways in which the letters on one display could possibly be grouped.

\section{Results}

Table 2 shows the percentage of time the target was grouped with the mask for each stimulus condition. At the close spacing, subjects perceived the target as being grouped with the mask more often in the single than in the group mask condition. These grouping data, then, do not support a perceptual grouping explanation of the close spacing results. Somewhat surprisingly, the subjects saw the target as being grouped with the mask about equally often in the single and grouped conditions at distant spacing. Using chisquare tests, the difference between the ratings at close spacing was not significant $(p=.44)$, but the difference approached significance with distant spacing $(p=.06)$. At $1 \mathrm{deg}$ of spacing, the subjects seldom judged the target as being grouped with the mask(s). We will return to this anomaly in the third experiment.

\section{EXPERIMENT 2A}

The second experiment was carried out to compare again the grouping and feature-interaction hypotheses using new stimulus configurations. Only relatively close target-mask spacing was used. The two stimulus configurations used (Figure 3) differ in the presence or absence of a mask letter on the same horizontal meridian as the target letter. The stimuli were chosen with the expectation that the target would be grouped with the mask letters more often in the 2-H, "wing," display than in the 3-H, "column," display. Based on that expectation, the grouping hypothesis would predict poorer letter-detection performance for the wing configuration. Due to the increased opportunity for featural interaction, feature interaction, however, predicts poorer performance in the configuration containing the mask directly peripheral to the target.

\section{Method}

Subjects. Sixteen Dartmouth introductory psychology students participated for course credit.

Stimuli. The target letters and mask letter were the same as those used in the first experiment. Figure 3 shows the "wing" and "column" stimulus configurations. Mask letters were always peripheral to the target, with .4 deg of horizontal spacing between the center of the target and the center of the line of masks. The spacing between target and masks was wider than we would have liked. Pilot data, however, led us to believe that at least that much separation was required to achieve the desired perceptual groupings. Targets were presented $6.0 \mathrm{deg}$ to the left or right of the centered fixation point for $100 \mathrm{msec}$. The apparatus was the same as in Experiment 1 .

Procedure. The procedure was similar to that used in the first experiment, with these exceptions: Subjects served in only one session, with eight blocks of 64 trials $(4$ target letters $\times 2$ left-right visual field $\times 8$ repetitions), preceded by four blocks of 16 practice trials using the same stimuli at the same duration. Stimulus configuration was blocked, with each subject receiving four blocks with each mask type. Feedback on performance was given after every two blocks. One second (rather than 2) elapsed between onset of the fixation cross and the onset of the stimulus.

\section{Results}

Accuracy on the wing stimulus was higher than accuracy on the column stimulus, $43.6 \%$ vs. $41.3 \%$ $[F(1,15)=4.48, p<.05]$, supporting the featureinteraction interpretation. In addition, 13 of $16 \mathrm{sub}$ jects performed better on the wing than on the column displays. This is significant using a sign test $(p=.022)$. There was a significant main effect of target letter. The letter $T$ was more discriminable than the other three targets $[F(3,45)=7.86, p<.0005]$. In addition, the interaction of visual field and display type (wing or column) reached significance $[F(1,15)=5.68$,

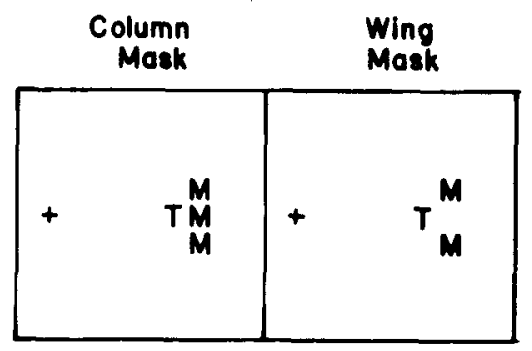

Figure 3. Stimulus configurations for Experiment 2. 
$\mathrm{p}<.05]$. This was due to the relatively greater superiority of wing stimuli vs. column stimuli in the right visual field than in the left visual field.

\section{EXPERIMENT 2B}

\section{Method}

Experiment 2B was carried out to collect ratings of grouping for the stimulus configurations used in Experiment $2 \mathrm{~A}$.

After completing the letter-detection task of Experiment $2 \mathrm{~A}$, each subject was given 16 trials (4 targets $\times 2$ stimulus configurations $\times 2$ visual fields) using the same stimuli as in Experiment $2 \mathrm{~A}$. The instructions and procedure were the same as in Experiment 1B. As in the earlier rating task, the subjects were instructed to write down what they saw and then circle the groupings they perceived. In addition, 16 subjects who had not been in Experiment $2 \mathrm{~A}$ served as subjects in this task. These new subjects allowed for a test of the effect of having served in the letter-detection task on perceived grouping.

\section{Results}

Ratings were classified into two disjoint categories: grouping the target with at least one mask or grouping the target separately. Subjects from Experiment 2A grouped the target with the mask $65 \%$ of the time for wing stimuli and $57 \%$ of the time for column displays (see Table 2). Subjects who had not been in Experiment $2 \mathrm{~A}$ grouped the target with the mask $59 \%$ of the time for wing stimuli and $54 \%$ of the time for the column stimuli. Pooling over all 32 subjects, the target was grouped with the mask $62 \%$ of the time for wing stimuli, and $54.5 \%$ of the time for the column stimuli. A two-factor analysis of variance on percentages of grouping targets with masks, with the two types of stimulus configurations and participation or nonparticipation in Experiment $2 \mathrm{~A}$ as factors yielded no significant differences among any factor or combination of factors (all Fs $<1$ ). Once again, the perceived groupings do not predict the accuracy data. At a descriptive level, the perceived groupings are opposite the accuracy measures.

As an additional test of the effect of grouping on performance, we compared the detection accuracy of subjects in Experiment $2 \mathrm{~A}$ as a function of whether they grouped the target with the mask or separately. The 16 letter-detection subjects were divided into two groups based on whether they grouped the target with the mask or separately the majority of the time. This breakdown was done separately for each stimulus configuration. For the wing stimuli, there were eight subjects in each group. The mean accuracy of the eight subjects who grouped the target with wing mask was $47.25 \%$. The mean accuracy of subjects who grouped the target and mask separately was about 7 percentage points lower, $40.0 \%$. For the column stimuli, nine subjects grouped the target with the mask consistently and seven grouped them separately. The mean accuracy of the nine subjects who grouped the target and mask of column stimuli to- gether was $43 \%$, and for those who grouped them separately, 39\%. Neither test was significant [for the wing stimulus, $t(14)=1.22$; for the column stimulus configuration, $t(14)=.61$ ]. For both stimulus configurations the performance of those subjects who perceived the target as grouped with the mask was slightly superior, contrary to a grouping hypothesis. These results argue against a perceptual grouping explanation of the accuracy of detection at the spacing used.

The results of the second experiment again show that a grouping explanation is not sufficient to explain the lateral masking effects at close spacings. The target was grouped with the mask more often in wing displays than in 3-H displays, yet performance was better on wing displays. The effects are small but quite consistent across subjects. The results are consistent with a feature-interaction explanation.

\section{EXPERIMENT 3}

Most of the literature reviewed in the introduction and the results of the first two experiments are consistent with the hypothesis that grouping effects (or distribution of attention) are good predictors of performance at wide spacing but that featural interactions dominate at close spacing. The perceived groupings in Experiment 1B at distant spacing, however, do not conform to the a priori assumptions of the experiment. At $1 \mathrm{deg}$ of spacing, the target was rarely grouped with the mask either in the single-mask displays or in the group-mask displays. There was even a tendency (not significant) to group the target less often with the mask in single-mask displays. The stimulus arrangements were chosen by Banks et al. (1979) based on Gestalt principles. The problem in the present case seems to be that subjects simply do not perceive much grouping between target and mask(s) at $1 \mathrm{deg}$ of separation.

If the perceived groupings do not predict performance, how, then, can we explain the superiority of grouped vs. single mask at distant spacing? One possibility is that the grouped mask serves as a cue to target location. Both in Banks et al. (1979) and in Experiment 1A there are two cues to target location aside from mask type. The first cue is the central/ peripheral dimension. During each block of 64 trials, the target is always central to or always peripheral to the mask(s) and subjects are informed about the assignment prior to the start of each block. Although this is a perfectly valid cue, it may lead to occasional confusion due to changes across blocks. The second cue is letter identity, since the mask is always the letter " $H$ " and the target one of four other letters. The trouble with this cue is that it requires letter identification.

In the grouped-mask condition of Experiment 1A, 
there is a third possible cue, namely, that the target is the one that there is not five of. This cue remains valid throughout the experiment and may be fairly salient. No similar cue is available in the single-mask displays.

We designed the third experiment as a test of the cue-validity hypothesis. The basic idea was to create a situation in which the presence of a column of five letters was not a reliable predictor of target or mask. To accomplish this, we used three groups. In all three groups, some of the blocks contained a single target and a single mask separated by a degree of visual angle. In the first group, the second set of blocks contained a single target and a column of five masks, as in Experiment 1A. In the second group, the second set of blocks contained a single mask and a column of five targets. In the third group, each of the second set of blocks contained some trials with one target and five masks and some trials with five targets and one mask.

The grouping hypothesis expressed by Banks et al. (1979) predicts an advantage for latter block types (i.e., grouped masks or grouped targets) in all three groups, since the grouping should be equally effective in isolating the target(s) from mask(s). The amount of improvement might vary as the presence of multiple targets in the two groups may lead to increments in addition to those attributable to grouping.

The cue validity hypothesis predicts little or no advantage for the grouped conditions of the third group. In the first two groups, a column of five letters is a perfect predictor of target location-negative in the first group and positive in the second group. In the third group, however, a column of five letters provides no information about target location. All three groups also had some blocks in which only the target was displayed. These blocks were included to assess whether masking was actually occurring in the grouped conditions.

\section{Method}

Subjects. Forty-eight undergraduates in an introductory psychology course, participating for course credit, were assigned to three groups of 16 each.

Stimuli. All subjects saw three types of stimulus displays, two of which were the same for all groups of subjects: target alone and single target plus single mask. The target-alone display was simply the target letter, uppercase $A, Y, U$, or T presented $5.23 \mathrm{deg}$ to the left or to the right of the fixation point. The single mask/ target displays were identical to the single mask displays of Experiment $1 \mathrm{~A}$, including both central and peripheral masks. Targetmask separation was always $.96 \mathrm{deg}$.

The third kind of stimulus display was different for each of the three groups of subjects. One group of subjects saw a groupedmask display identical to the grouped-mask display of Experiment 1. A second group of subjects saw a grouped-target display, with the target letter repeated five times in a vertical column centered at the target location (5.23 deg peripheral to the fixation cross, as before) and single-mask letter $\mathrm{H}$ at $.96 \mathrm{deg}$ away (spacings for this condition were identical to those in the grouped-mask condition). A third group of subjects saw both of these types of displays, either a grouped-mask or a grouped-target display on a given trial, randomly intermixed within a block with equal numbers of each type of display.
Procedure. Stimuli were presented on the same apparatus as in the previous experiments. The duration of stimulus presentation and of the intertrial interval was the same as in Experiment $2 \mathrm{~A}$.

The subjects received instructions similar to those in Experiments $1 \mathrm{~A}$ and $2 \mathrm{~A}$. They were also instructed to look at a list posted beside them that explained the position of the target letter(s), in relation to the mask letter(s), for each block of trials. These lists were, of course, different for each of the three groups of subjects.

The subjects then received five blocks of practice trials, with 16 trials in each block, corresponding to the five mask conditions: target alone (no mask), single central mask, single peripheral mask, and central and peripheral grouped displays of the three types listed above, grouped-target or grouped-mask or both, depending on the subject's assignment to the groups. These trials were identical to the experimental trials that followed.

The subjects received 10 blocks of experimental trials, with 32 trials in each block, 2 blocks for each of the five mask conditions. The order of blocks was counterbalanced within each subject's session. All targets were presented equally often, and randomly ordered, as before. Most subjects completed the experiment within $45 \mathrm{~min}$.

\section{Results}

Several analyses were carried out. First we carried out an analysis of variance on all of the data. There were four factors: visual field, letters, block type (target alone, single central mask, single peripheral mask, group central, and group peripheral), and group assignment (multiple targets, multiple masks, and mixed). As usual, the effect of letters was highly significant, with $T$ being more easily detected than the other letters. The effect of block type was significant $[F(4,180)$ $=14.06, p<.01]$. This effect resulted primarily from the superiority of the target-alone conditions.

Of primary interest was a significant interaction between block type and group assignment $[F(8,180)$ $=4.25, \mathrm{p}<.01]$. This interaction is illustrated in Figure 4. As shown in Figure 4, in the group with multiple masks only, performance with multiple masks was superior to performance with a single mask. This superiority of masks replicates Experiment 1A (with far spacing) and Banks et al. We examined the significance of this increase by carrying out an analysis of variance on just those subjects in the multiple masks group. There were three factors: visual field, letters, and block type. The effect of letters was significant. The effect of block type was also significant $[F(4,60)=5.16, p<.01]$. A planned comparison revealed that performance on the grouped-mask conditions was superior to performance on the singlemask conditions $[\mathrm{F}(1,60)=5.03, \mathrm{p}=.027]$.

Returning to the interaction depicted in Figure 4, in the group with multiple targets only, performance with multiple targets was superior to performance with a single target. Again, we carried out an analysis of variance on just those subjects in the multipletargets group. The effect of letters was significant. The effect of block type was highly significant $[F(4,60)$ $=9.92, \mathrm{p}<.01 \mathrm{l}$. A planned comparison revealed that performance on the grouped target displays was superior to performance on the single-target displays $[F(1,60)=30.01, p<.01]$. 


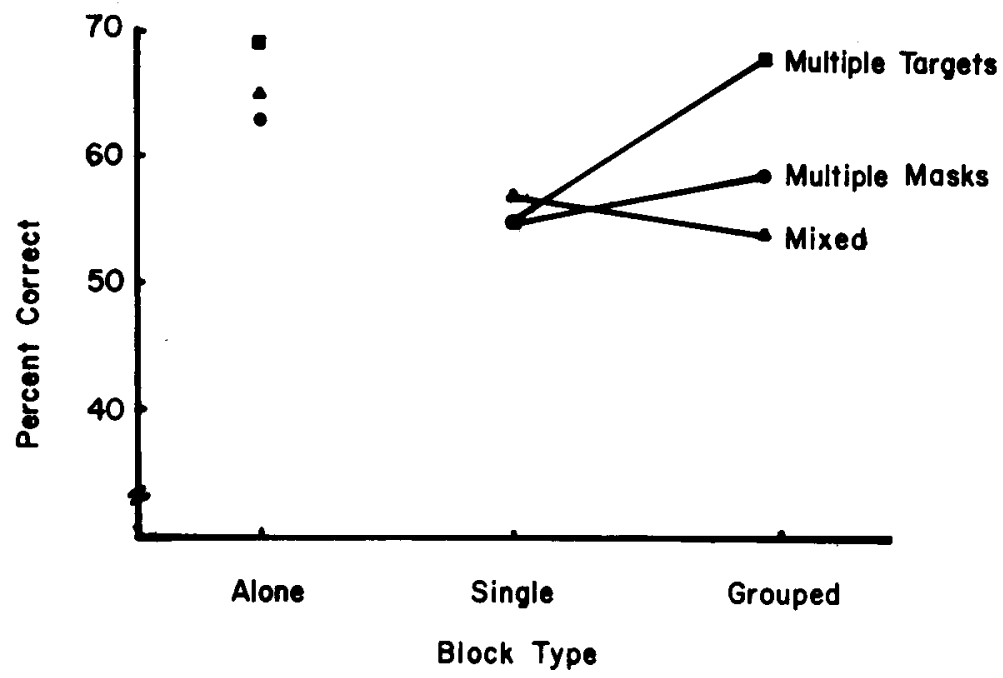

Figure 4. Performance in Experiment 3 as a function of grouping and cue value.

Finally, in Figure 4, in the group with mixed multiple targets and multiple masks, performance on the grouped displays was slightly worse than performance on the single displays. In a separate analysis of variance on this group, the effect of block type was significant $[F(4,60)=5.50, p<.01]$. A planned comparison revealed that performance on the grouped displays was not significantly different from performance on single displays $[F(1,60)=1.68, p=.20]$. The main effect of block type reported above was attributable to the superiority of the target-alone condition.

Finally, in this last group with mixed multiple targets and masks, we examined performance on the two halves of the mixture. For the grouped displays, on the half of the trials on which multiple targets were displayed, subjects responded correctly $54.8 \%$ of the time; on the half of the trials with multiple masks, subjects responded correctly $54 \%$ of the time. This difference did not approach significance $(F<1)$.

The central vs. peripheral placement of mask(s) did not lead to any main effects or interactions in this experiment. Averaged across groups, it was true, however, that peripheral masks led to slightly lower performance on the single displays while central masks led to slightly lower performance with grouped masks, as in Banks et al. This effect did not even approach significance in our experiment.

What do the results of Experiment 3 tell us about the effect of grouping? Banks et al. (1979) argued that perceptual grouping achieved its effect by perceptually isolating the target from the mask. We believe that grouping achieved its effect by providing information about target location. When that information is eliminated (as in the mixed group of this experiment), the effect of grouping disappears. Our explanation in terms of cue validity is not radically different from that of Banks et al., as our explanation subsumes a perceptual grouping hypothesis. Subjects must perceive the grouping to take advantage of any location information. The difference between our explanations is that we do not believe that the perception of grouping inherently leads to superior performance on a particular trial. The perception of grouping facilitates performance only when that information is correlated across trials with target information.

\section{GENERAL DISCUSSION}

The results of the three experiments support the contention that lateral masking has qualitatively different properties at close and distant spacing. At close spacing, the results are consistent with an interaction between target and mask at the featural level. The comparison of Kahneman and Henik (1977) with Wolford and Hollingsworth (1974), the results of Experiment $1 \mathrm{~A}$ at close spacing, and the results of Experiment 2A all show that something like feature interaction dominates grouping effects at close spacing. These close-spacing results are consistent with the general class of featural models outlined in the introduction. The results, however, do not distinguish among specific accounts of contour interaction.

At wider spacing, the effects of lateral masking are inconsistent with a featural interpretation and appear to be related to the distribution of attention. According to this interpretation, observers have some control over the distribution of attention during brief glances and the items which receive the bulk of the attention are most accurately reported. Several factors, such as grouping, the number of items within a group 
(see Kahneman \& Henik, 1977), and spatial location, appear to control the distribution of attention. The results of the third experiment suggest that some of the effects of grouping may be in the information provided by the groups as to the location of the target.

A lateral mask, then, influences performance in several ways. It can draw some of the observer's attention away from the target, and, if the spacing is close enough, the mask and target appear to interact at a featural level, further disrupting performance. The lateral mask can further influence performance if it is drawn from the set of possible responses. As the work of Eriksen and his colleagues, summarized in the introduction, has shown, masks from a compatible response set facilitate performance and masks from an incompatible response set hinder performance. All of these variables need to be included in a comprehensive account of lateral masking.

\section{How Close is Close?}

Throughout this research we have used the term close spacing. Over what retinal separation will two stimuli interact at the feature level? The answer appears to depend on the retinal eccentricity of the target. Near the center of the fovea, features appear to interact only over very short separations. Further into the periphery, characters interact over a wider separation.

A number of studies have varied target-mask separation. It is not generally clear how to relate the results to feature interaction, since we argue that lateral masking is a composite of several variables. In one of the clearest studies, Flom, Weymouth, and Kahneman (1963) presented targets at the center of the fovea and varied target-mask separation over a number of small steps. They found rather steep functions, with the mask interfering with the target only if they were separated by $.1 \mathrm{deg}$. C. W. Eriksen and Hoffman (1972) presented targets 2 deg into the periphery. They found considerable masking at $.5 \mathrm{deg}$ but little or none from $1.0 \mathrm{deg}$ on. Bouma (1970) orthogonally varied the retinal eccentricity of the target and the target-mask separation. He estimated that the range over which target and mask interact was about one half of the target eccentricity. Some of the interaction in Bouma's study, however, may have resulted from the nonfeatural components of lateral masking. If so, his figures are probably an overestimate of the range of feature interactions.

There is some suggestive work from the data on receptive field size. Hubel and Wiesel (1977) estimated that aggregate receptive fields in monkeys were $.1 \mathrm{deg}$ in diameter at the center of the fovea and $.5 \mathrm{deg}$ in diameter $7 \mathrm{deg}$ into the periphery. An aggregate receptive field is the combined receptive field of all of the cells in a single column of the visual cortex. It is possible that two stimuli interact at the feature level if they fall in the same aggregate receptive field. Fuld (1978) used the Westheimer effect to estimate cortical receptive field size in humans. Based on his data, he estimated that receptive fields in the center of the fovea had an area of $.08 \mathrm{deg}$ and those $2.5 \mathrm{deg}$ into the periphery, an area of $.66 \mathrm{deg}$. These various estimates of receptive field size are in the same range as the estimates of the extent of lateral masking summarized above.

Santee and Egeth (1982) recently reported an experiment in which they found no effect of letter separation. Although these data appear to contradict our conclusions, they are potentially quite consistent. Santee and Egeth varied intercharacter spacing by presenting the letters on circles of different diameters. As a result of this technique, letter separation covaries with retinal eccentricity. Since we suggest that the distance over which two letters interact is a function of retinal eccentricity, it would be quite possible to choose a joint function of the two that yields no effect of letter separation.

\section{REFERENCES}

Banks, W. P., \& Prinzmetal, W. Configurational effects in visual information processing. Perception \& Psychophysics, 1976, 19, 361-367.

Banks, W. P., Larson, D. W., \& Prinzmetal, W. Asymmetry of visual interference. Perception \& Psychophysics, 1979, 25, 447-456.

BJORK, E. L., \& MURRAY, J. T. On the nature of input channels in visual processing. Psychological Review, 1977, 84, 472-484.

Boums, H. Interaction effects in parafoveal letter recognition. Nature, 1970, 226, 177-178.

BoumA, $H$. Visual interference in the parafoveal recognition of initial and final letters of words. Vision Research, 1973, 13, 767.782.

Chastain, G., \& Lawson, L. Identification asymmetry of parafoveal stimulus pairs. Perception \& Psychophysics, 1979, 26, 363-368.

Eriksen, B. A., \& Eriksen, C. W. Effects of noise letters upon the identification of a target letter in a nonsearch task. Perception \& Psychophysics, 1974, 16, 143-149.

Eriksen, C. W., \& Hofrman, J. E. The extent of processing of noise elements during selective encoding from visual displays. Perception \& Psychophysics, 1973, 14, 155-160.

Eriksen, C. W., \& Rohrbaugh, J. W. Some factors determining efficiency of selective attention. American Journal of Psychology, $1970,83,330-342$.

Ehiksen, C. W., \& Schultz, D. W. Temporal factors in visual information processing. In J. Requin (Ed.), Attention and performance VII. Hillsdale, N.J: Erlbaum, 1978.

Estes, W. K. Interactions of signal and background variables in visual processing. Perception \& Psychophysics, 1972, 12, 278-286.

Flom, M. C., Weymouth, F. W., \& Kahneman, D. Visual resolution and contour interaction. Journal of the Optical Society of America, 1963, 53, 1026-1032.

FuLD, K. A sensitization effect with rectilinear stimuli. Vision Research, 1978, 18, 1045-1051.

Hubel, D. H., \& Wiesel, T. N. Ferrier lecture: Functional architecture of macaque monkey visual cortex. Proceedings of the Royal Society of London (Series B), 1977, 198, 1-59.

Kahneman, D., \& Henik, A. Effects of visual grouping on immediate recall and selective attention. In S. Dornic (Ed.), Attention and performance VI. Hillsdale, N.J: Erlbaum, 1977. 
Kole rs, P. A., \& Rosner, B. S. On visual masking (metacontrast): Dichoptic observation. American Journal of Psychology, $1960,73,2-21$.

KrumhansL, C. L. Naming and locating simultaneously and sequentially presented letters. Perception \& Psychophysics, 1977, 22, 293-302.

Mackworth, N. H. Visual noise causes tunnel vision. Psychonomic Science, 1965, 3, 67-68.

MatThews, M. L. Locus of presentation and the selective masking effect. Canadian Journal of Psychology, 1973, 27, 343-349.

Prinzmetal, W. Principles of feature integration in visual perception. Perception \& Psychophysics, 1981, 30, 330-340.

SanteE, J. L., \& Egeth, H. E. Independence versus interference in the perceptual processing of letters. Perception \& Psychophysics, 1982, 31, 101-116.

Treisman, A., \& Schmidt, H. Illusory conjunctions in the perception of objects. Cognitive Psychology, 1982, 14, 107-141.

Wolfond, G. Perturbation model for letter identification. Psychological Review, 1975, 82, 184-199.

Wolford, G., \& Holdingsworth, S. Lateral masking in visual information processing. Perception \& Psychophysics, 1974, 16, 315-320.

(Manuscript received June 18, 1982;

revision accepted for publication November 5,1982 .) 